\title{
Interfaz de Consulta con Lenguaje Python para el Catastro de Redes de Acueducto
} y Alcantarillado

\section{Querying Interface with Python Language for The Cadaster of Aqueduct and Sewer Networks}

\author{
Diana Elizabeth Ortiz Carvajal ${ }^{1}$ \\ https://orcid.org/0000-0002-6750-2705 \\ Diego Iván Sánchez Tapiero ${ }^{2}$ \\ https://orcid.org/0000-0002-7270-1813 \\ Universidad de Pamplona, Colombia
}

Recibido:15-05-2021

Aceptado: 30-08-2021

\section{Cita Recomendada}

Ortiz, D. y Sánchez, D. (2021). Interfaz de consulta con lenguaje Python para el catastro de redes de acueducto y alcantarillado. Hamut' ay, 8 (2), 79-97, http://dx.doi.org/10.21503/hamu.v8i2.2295

\section{Resumen \\ En Ingeniería Civil, la disposición de un apropiado catastro técnico de la infraestructura de acueducto y alcantarillado, es un instrumento esencial para la eficaz operación y el correcto mantenimiento de estas redes, con el propósito de mejorar el buen funcionamiento y optimizar los recursos de este tipo de empresas, como práctica necesaria dentro de sus planes de mejoramiento. Es por ello que se hace necesario el implementar modelos de vanguardia tecnológicos, como los que posibilita el lenguaje de programación Python, con el que se puede crear una interfaz de consulta catastral para el acceso a información catastral de manera rápida, precisa, oportuna, eficaz, ordenada y simplificada, con miras a optimizar su gestión y almacenamiento. Esto plantea el organizar, computarizar y actualizar el inventario físico de estas redes, mediante la creación de una herramienta de consulta permanente, que registre datos confiables y oportunos para la futura toma de decisiones. \\ El objetivo principal del catastro de redes en el presente estudio, es contar con una base de datos técnica debidamente geo-referenciada en el software QGIS que proporcione información real y detallada de los sistemas existentes en el municipio de Chitagá (Google Earth, 2021), y así contar con un inventario veraz y confiable sobre las redes de acueducto y alcantarillado, como referente para la toma de decisiones; la gestión de estas redes; el planteamiento de planes de mejora; el monitoreo, la optimización y la ampliación de servicios, además de convertirse en un indicador del nivel de calidad, progreso, modernidad y actualización del municipio y de igual manera, en sinónimo de prácticas satisfactorias de investigación ingenieril.}

Palabras Clave: Python, Acueducto, Alcantarillado, Catastro, Sistema De Información Geográfica QGIS, Interfaz.

1. Semillero AQUA, Grupo de Investigación ETENOHA; Estudiante de Ingeniería Civil; diana.ortiz2@unipamplona.edu.co, dianaortizcc2014@gmail.com.

2. Director de la Tesis de Investigación; Coordinador del Semillero AQUA, Grupo de Investigación ETENOHA del Programa de Ingeniería Civil, Universidad de Pamplona; Ingeniero Civil; diego.sanchez@unipamplona.edu.co. 


\begin{abstract}
In Civil Engineering, the availability of an appropriate technical cadaster for the aqueduct infrastructure is an essential instrument for the effective operation and maintenance of these networks, with the purpose of improving the proper functioning and optimizing the resources of this type of companies as a necessary practice within their improvement plans. That is why it is necessary to implement cutting-edge technological models, such as those made possible by the Python programming language, in which a cadastral query interface can be created to access cadastral information in a fast, precise, timely, and efficient manner, organized and simplified, with the aim of optimizing its management and storage. This raises organization, digitization and updating of the physical inventory of these networks, through the creation of a permanent consultation tool that records reliable and timely data for future decision-making.

The main objective of the network registry in this study is to have a technical database duly geo-referenced in the QGIS software that provides real and detailed information on the existing systems in the municipality of Chitagá, and thus to have a truthful and reliable inventory on the aqueduct and sewerage networks, as a reference for decision-making; the network management; the elaboration of improvement plans; the monitoring, optimization and expansion of services, in addition to becoming an indicator of the level of quality, progress, modernity and renewal of the municipality and in the same way, synonymous with satisfactory engineering research practices.
\end{abstract}

Key words: Python, Aqueduct, Sewerage, Cadaster, QGIS Geographic Information System, Interface.

\section{Introducción}

El saneamiento básico ha sido una necesidad primordial que data desde tiempo atrás. En la actualidad, existen considerables requerimientos técnicos para mantener el bienestar y la salud de las localidades, necesidades que dependen de la construcción, administración y diseño de los recursos e infraestructuras que conforman la red y sus suscriptores (Departamento Nacional de Planeación Financiera de Desarrollo Territorial, 1997).

Los antecedentes que se detallan a continuación muestran esta preocupación por el saneamiento. Solano, (2002) señala que la dirección de una red de distribución de agua potable requiere información sobre datos físicos de los elementos, datos económicos del sistema y datos espaciales sobre la ubicación de los componentes, así como la existencia de un adecuado SIG (Sistema de Información Geográfica). Por su parte, Sánchez y Amorós (2012) adelantaron un paso en el desarrollo de esta nueva tecnología, además de utilizarla para realizar consultas y hacer mapeos, también analizaron tendencias $y$ tomaron decisiones mediante las diferentes aplicaciones que ofrecen los análisis espaciales.
Fragoso, Ruiz y Juárez (2013) describen un proyecto que implantaron en un SIG en la gestión de información espacial y no espacial de una red de distribución de agua potable de dos unidades habitacionales en Tehuacán, Puebla, México, para que el beneficiario visualice y efectúe operaciones con la información de una base de datos.

Avendaño y Cadena, (2014) en una investigación cuantitativa, aplicaron una herramienta de información cartográfica para representar las áreas con mayor vulnerabilidad de inundación a los organismos de control, dando paso a la generación de medidas de prevención y mitigación en temporada de lluvias. Asimismo, Diaz y Mercado (2016), presentaron un análisis de la red de agua en el municipio de San Juan Nepomuceno, arrojando a través de la implementación de SIG y el uso de la herramienta WaterGEMS V8I for ArcMap, variables principales como el caudal, la presión del agua, la topografía y los niveles de velocidad. Muñoz, Roncancio y Mendoza (2018), en su trabajo aplicado dentro del software ArcGis, otorgaron a la Armada Nacional de Colombia un instrumento poderoso tanto para el funcionamiento físico, administrativo y operativo, como para la evaluación de las 
Plantas de Tratamiento de Agua Potable y Residual (PTAR), a partir de los resultados de pruebas fisicoquímicas y biológicas. De igual manera Jaimes (2019), desarrolló un sistema de información geográfica sobre la plataforma ArcGIS, para el manejo de datos en forma digital del catastro de la red de alcantarillado. Además, en el modelo de cálculo hidráulico e hidrológico del software SWMM, entregó la simulación de la red de alcantarillado combinado en condiciones ideales, lo que llevó a conocer los niveles de la lámina de agua en cada uno de los colectores y pozos del sistema. Buitrago (2018), organizó la información existente en un Sistema de Información Geográfica (SIG), con el fin de manejar y gestionar la información para la toma de decisiones de los administradores.

Los anteriores antecedentes, muestran la importancia que tiene para los municipios el que las empresas de servicios cuenten con SIG, que les permitan recopilar, organizar, registrar, almacenar, actualizar, analizar, evaluar y desplegar información acerca del funcionamiento de los sistemas de acueducto y alcantarillado, que contribuyan a su fortalecimiento y gestión.

Es así que el presente estudio se enfocó en el desarrollo de una propuesta de implementación de un SIG para el catastro delas redes de acueducto y alcantarillado del municipio de Chitagá (Norte de Santander), con el propósito de mejorar los índices de calidad en la prestación de servicios y proveer la optimización del sistema. Como objetivos específicos se plantearon: (1) Actualizar el levantamiento topográfico y el catastro técnico de las redes de acueducto y alcantarillado para el reconocimiento del terreno del municipio y la infraestructura que compone estos sistemas. (2) Construir un diccionario de datos que integre toda la información recolectada en campo derivada del catastro de redes con metodologías y procedimientos adecuados que permita observar el estado actual de los sistemas. (3) Incorporar la información recolectada en el SIG para la toma de decisiones futuras en cuanto a disponibilidades, reparaciones, ampliaciones, coberturas de nuevos sectores y planes de optimización y mejoramiento. (4) Programar sentencias en código Python tanto para la intervención de los sistemas de redes de acueducto y alcantarillado como la evaluación de su funcionamiento. (5) Generar estrategias y seguimiento de control en los sistemas de acueducto y alcantarillado, mediante consultas rápidas desde los sistemas de información geográfica. (6) Simular el funcionamiento de las redes de acueducto y alcantarillado existentes mediante los softwares EPANET y SWMM para la obtención de las condiciones mínimas de diseño de una manera más rápida y sencilla. (7) Diseñar en código Python la interfaz de consulta que integre la información producto del catastro de redes.

\section{Sistemas de Información Geográfica (SIG)}

Muchas son las definiciones sobre un SIG, unas más perfeccionadas que otras, pero todas resaltan dos particularidades básicas del SIG: (a) Es un sistema administrador de bases de datos que se encuentran georreferenciados. (b) Guarda e integra datos espaciales de posición o localización, con datos temáticos (atributos alfanuméricos) divididos en diferentes coberturas o capas.

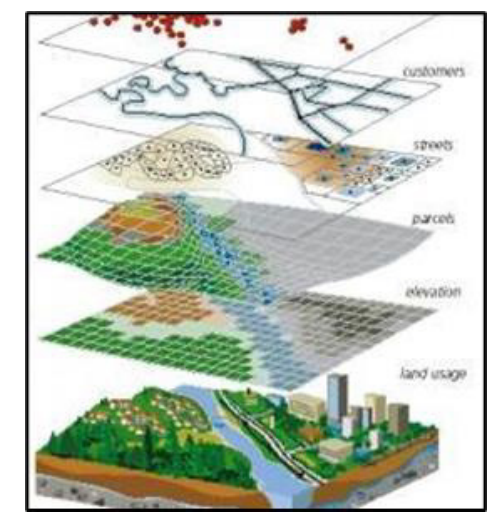

Figura 1. Representación del entorno en un SIG disposición de capas de información.

Fuente: (Biere y Aitziber, 2010)

Según Biblioteca Virtual, Luis Ángel Arango, 2021 "la información espacial se almacena y se procesa a manera de puntos, redes, líneas, polígonos y áreas a las cuales se le otorgan valores que pueden ser geo-referenciados por el programa”. 
De acuerdo con (Borcosque, 1997), la implementación de los sistemas de información geográfica en una organización trae consigo la mejora en el ordenamiento de los datos referenciados espacialmente. Por esta razón, (Healey, 2001) los Sistemas de Información Geográfica (SIG) son una herramienta idónea de análisis de información, están diseñados para trabajar tanto con datos referenciados (coordenadas) como con bases de datos. (Gulf of California Marine Programa, 2021).

La primera aproximación al uso de los SIG en la gestión de redes de distribución de agua potable y recolección de aguas residuales documentada en la bibliografía se produce en Denver Waters (Cesario, 1986).

El catastro de redes es una actividad considerada de corta duración, lo que facilita el restablecimiento de las condiciones de normalidad de la zona intervenida. (EPM, Version 08, 2021, p. 81). En lo relacionado con Sistemas de Información Geográfica destacan los aportes de ESRI, (2016); Gobernación de Norte de Santander- DAG Ingeniería, (2014, p. 32); Gulf of California Marine Programa, (2021), IGAC, (1998); Rossiter, (1994); Zarzosa y Andrés, (2004); Gómez, Velasquez y Rodriguez, (2006), Guzmán, (2021), Hernández y Osorio, (2019); López, (2012), Morales y Rodríguez, (2016); Moran y Malma, (2013); Palacio, (2017); Rodríguez, A. y Rodríguez, D. (2014); Santiago, (2010); Sarría, (2010); Torres, (2015), Zapata, Martinez y Gonzalez, (s.f.); Zarzosa y Andrés, (2004).

\section{QUANTUM GIS (QGIS)}

En el manejo de información geográfica se empleó el software QUANTUM GIS (QGIS) versión 3.10, como un "sistema que permite recopilar, organizar, administrar, analizar, compartir y distribuir información geográfica, para poner al servicio de los sectores del gobierno, la empresa, la ciencia, la educación y los medios el conocimiento geográfico" (Orjuela, 2018). QGIS, es un software de código abierto que hace referencia a direcciones, lugares, ubicación de terrenos en áreas urbanas y rurales, regiones y cualquier tipo de ubicación o posición.

Según Avendaño y Cadena, (2014) las características principales de QGIS son el soporte a tablas de datos no espaciales, a datos ráster o vectoriales, a WMS y WFS, además de la digitalización, edición e impresión de información. Y el SIG usado con mayor frecuencia es ArcGis (ESRI, 2016).

\section{Reglamento del agua potable}

Para el desarrollo del SIG, se tomó como referencia el Reglamento Técnico de Agua del Sector de Agua Potable y Saneamiento Básico RAS (Ministerio de Vivienda, 2017), en el que se señalan los requisitos que deben cumplir las obras, los equipos y los procedimientos operativos utilizados en la prestación de los servicios públicos domiciliarios de acueducto, alcantarillado y aseo. En esta reglamentación "se define al catastro de redes como el registro de todos los elementos existentes en el sistema, con metodologías y procedimientos apropiados de campo". En el artículo $8^{\circ}$ de la Resolución 0330 del 08 de junio de 2017 (Ministerio de Vivienda, 2017) se adopta el RAS y se indica la necesidad de contar con un CATASTRO DE REDES, el cual, en esta propuesta, es gestionado mediante el manejo de información espacial a través de un software especializado.

\section{Python}

Python fue creado a finales de los años 1980 por Guido van Rossum en el centro CWI de Ámsterdam. "Es un lenguaje de programación multiparadigma, ya que soporta parcialmente la orientación a objetos, programación imperativa $y$, en menor medida, programación funcional. Es un lenguaje interpretado, dinámico $y$ multiplataforma" (Pérez, 2014). Dado que su programación es sencilla, los tiempos de desarrollo son más cortos, los programas cuentan con menos líneas de código, son más fáciles de leer y se facilita la detección de errores. Así mismo, al ser un lenguaje interpretado, puede ser 
utilizado en cualquier computador donde exista un intérprete.

\section{EPA SWMM}

El EPA SWMM Stormwater Management Model (modelo de gestión de aguas pluviales), "es un modelo dinámico de simulación de precipitaciones, que se puede utilizar para un único acontecimiento o para realizar una simulación continua en periodo extendido. El programa permite simular tanto la cantidad como la calidad del agua evacuada, especialmente en alcantarillados urbanos" (Martínez, 2013, p. 44). El módulo de transporte o hidráulico SWMM, analiza el recorrido del agua, junto con otros elementos como canales, bombas, elementos reguladores, dispositivos de almacenamiento y tratamiento, además de ser "capaz de seguir la evolución de la cantidad y la calidad de agua de escorrentía de cada cuenta, así como el caudal, el nivel de agua en los pozos o la calidad de agua en cada tubería y canal durante una simulación compuesta por múltiples intervalos de tiempo" (Rodríguez, A. y Rodríguez, D. 2014, p. 17).

\section{EPANET}

EPANET es un software de libre distribución creado por U.S. EPA, que permite la simulación del comportamiento hidráulico y la evaluación de la calidad del agua en redes de tuberías a presión, a partir de la concentración de sustancias en la red de distribución, la incorporación tiempos de simulación, el seguimiento de la presión en los nodos y el flujo en los conductos, así como la incorporación de su respectiva demanda y el nivel del agua en los depósitos. "Este programa ha sido diseñado como una herramienta de investigación para mejorar el conocimiento del movimiento y evolución de los componentes del agua en el interior de los sistemas de distribución" (Torres y Vélez, 2010, p. 27).
Sistema de Acueducto, de Alcantarillado y Catastro de redes

Etimológicamente la palabra acueducto significa "conducción de agua" (SENA, 2005) y su propósito principal es captar el agua de una fuente hídrica, transportarla hasta una planta de tratamiento de agua potable, tratar sus características fisicoquímicas, llevarla a tanques de almacenamiento, conducirla mediante una red y distribuirla a todos los habitantes suscritos al servicio de agua potable. Según Cualla (1995), un sistema de acueducto está conformado por la infraestructura necesaria para suministrar agua potable a una comunidad, las obras hidráulicas para la captación, el sistema de purificación del agua, la conducción, el almacenamiento y la distribución. Por otro lado, el sistema de alcantarillado es el conducto por el cual fluyen tanto el agua lluvia como las aguas negras (IGAC, 2004).

Como complemento a los sistemas de acueducto y alcantarillado, aparece el catastro de los sistemas de redes de distribución de agua potable $\mathrm{y}$ de transporte de aguas residuales, que registra y archiva información técnica como fotos, fichas técnicas y planos, la cual corresponde a todos los detalles técnicos de georreferenciación de cámaras de inspección, tuberías, hidrantes, válvulas, diámetros y todo lo relacionado a accesorios importantes que hagan parte de las redes o esté incorporado. Por ello, Sabogal y Rincón (2017) enfatizan que el catastro de redes es una herramienta esencial que posibilita unificar la información existente en un sistema y obtener el estado o el diagnóstico de posibles.

\section{Materiales y métodos \\ Participantes}

La población en la que se realizó el estudio fue el municipio de Chitagá, departamento Norte de Santander. Como muestra de investigación se tomaron las redes de los sistemas de acueducto y alcantarillado. El tipo de muestreo fue aleatorio simple en el que a cada elemento de la red se le hace el estudio, ya que es posible la reducción de 
las áreas a variables que toman un valor fijo en geometrías simples (puntos, líneas o superficies). En este modelo los datos son clasificados de acuerdo a su naturaleza y almacenados como objetos.

\section{Instrumentos}

La información existente se obtuvo por medio de una entrevista realizada al personal de las secretarías de Planeación y Servicios Públicos de la Alcaldía del municipio de Chitagá. La información en campo se recopiló con la ayuda de una calculadora Texas Inspire CX CAS en la que se crearon Formatos para el Diligenciamiento de los Datos en Campo (Figura 2). Una vez tomados los datos, al finalizar la jornada diaria se digitaron junto con las fotos Geo-Referenciadas en las Fichas Técnicas de Catastro (Figura 3).
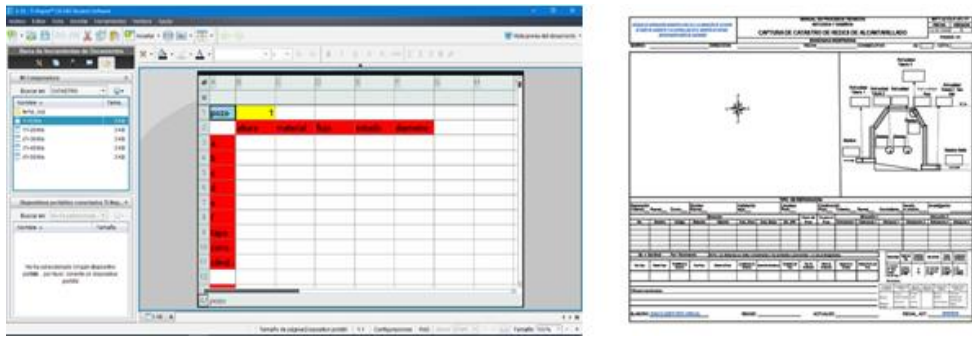

Figura 2. Instrumentos de Recolección de Información. Fuente: Elaboración propia (2021).

\section{Procedimiento}

El estudio se llevó a cabo a través de ocho fases iniciando en el mes de septiembre del año 2020 hasta los primeros 3 meses del año 2021, tiempo en el que se llevó a cabo el procedimiento para implementar Sistemas de Información Geográfica en la Elaboración de Catastro de Redes de Acueducto y Alcantarillado Municipio Chitagá, Departamento Norte de Santander (Tabla 1).
Tabla 1 Fases Metodología.

\begin{tabular}{l}
\multicolumn{1}{c}{ Fases de Investigación } \\
\begin{tabular}{l} 
1. Recopilación de Información Existente \\
\hline 2. Recolección de Información en Campo: \\
Planeación, Fichas Técnicas, Catastro de \\
Redes
\end{tabular} \\
\hline 3. Actualización Planos AUTOCAD \\
\hline 4. Diccionarios de Datos \\
\hline 5. Bases de Datos \\
\hline 6. Implementación del SIG QGIS \\
\hline 7. Diseño de Consultas Rápidas \\
\hline 8. Simulación de las Redes en Software: \\
SWMM, EPANET \\
\hline 9. Diseño de Interfaz Gráfica Python \\
\hline
\end{tabular}

\section{Confidencialidad o Consentimiento informado}

El estudio obtuvo el permiso por parte del jefe de Catastro del municipio de Chitagá, departamento Norte de Santander.

Resultados de la propuesta "Interfaz de consulta con lenguaje Python para el catastro de redes de acueducto y alcantarillado". A continuación, se describen los resultados obtenidos en cada una de las fases de la propuesta de la interfaz: (1) Recopilación de información existente. (2) Recolección de Información en Campo. (3) Actualización Planos AUTOCAD. (4) Diccionarios de Datos. (5) Bases de Datos. (6) Implementación del SIG QGIS. (7) Diseño de Consultas Rápidas. (8) Simulación de las Redes en Software: SWMM, EPANET. (9) Diseño de Interfaz Gráfica Python.

\section{Fase 1: Recopilación de información existente}

La recopilación de la información existente se llevó a cabo en una visita en la que se entrevistó al personal de la Alcaldía del municipio de Chitagá en las secretarías de Planeación y Servicios Públicos; quienes proporcionaron información del catastro de la red de alcantarillado del año 2017 con planos AutoCAD, cabe aclarar que sin referenciar. En cuanto a la red de acueducto 
dichas secretarías contaban solamente con un archivo .DWG del año 2012 y con planos en formato físico realizados en el 2016. Además, proporcionaron personal de fontanería para realizar las visitas de campo a cada uno de los pozos de inspección del sistema de alcantarillado. Se detectó, que el municipio no contaba con información unificada en cuanto a las redes y esto dificultó el obtener en primera instancia datos importantes, ya que no existía un proceso organizado de información. Reconocido el problema, se hace la propuesta de crear un sistema digital en el que se pueda acceder a la información, realizar consultas fáciles y rápidas sobre la base de datos, además de evitar que la gestión municipal presente una distorsión en el seguimiento del funcionamiento y control de los sistemas en estudio, con el fin de permitir una planeación efectiva de los recursos.

Tabla 2 Diccionario de Datos Tuberías Alcantarillado

\begin{tabular}{|c|c|c|c|}
\hline Id & $\mathbf{N}$ & $\begin{array}{l}\text { Pozo al que pertenece } \\
\text { la tubería domiciliaria }\end{array}$ & Pozo de entrada \\
\hline Pozo de salida & $\begin{array}{c}\text { Caracterización en el } \\
\text { pozo de entrada }\end{array}$ & $\begin{array}{l}\text { Caracterización en el } \\
\text { pozo de salida }\end{array}$ & Diámetro \\
\hline Material & $\begin{array}{l}\text { Prof. de la tubería en } \\
\text { el pozo de entrada }\end{array}$ & $\begin{array}{l}\text { Prof. de la tubería en } \\
\text { el pozo de salida }\end{array}$ & $\begin{array}{l}\text { Cota clave en el } \\
\text { pozo de entrada }\end{array}$ \\
\hline $\begin{array}{l}\text { Cota batea en el } \\
\text { pozo de entrada }\end{array}$ & $\begin{array}{l}\text { Cota clave en el } \\
\text { pozo de salida }\end{array}$ & $\begin{array}{l}\text { Cota batea en el } \\
\text { pozo de salida }\end{array}$ & $\begin{array}{c}\text { Dirección del } \\
\text { flujo domiciliarias }\end{array}$ \\
\hline $\begin{array}{l}\text { Dirección flujo } \\
\text { colectores }\end{array}$ & Longitud & Pendiente & Tipo de vía \\
\hline
\end{tabular}

Nota: Debido a la extensión de las tablas no es posible anexarlas. Se presenta la información que esta contiene.

\section{Fase 2: Recolección Información en Campo}

La importancia de esta fase radica en la definición de las herramientas necesarias para obtener un diagnóstico acertado, además de realizar la integración de la información a fin de poder llevar a cabo la investigación. En esta fase se planificó y se planteó el catastro. En la planeación se diseñaron las fichas técnicas de catastro en formato XLXS para cámaras o pozos de inspección, válvulas e hidrantes. Con la ayuda de la calculadora Texas Inspire CX CAS se crearon Formatos para el Diligenciamiento de los Datos en Campo, después de tomados estos datos al finalizar la jornada diaria se digitaban junto con las fotos Geo-Referenciadas en las Fichas Técnicas de Catastro (Figura 3).

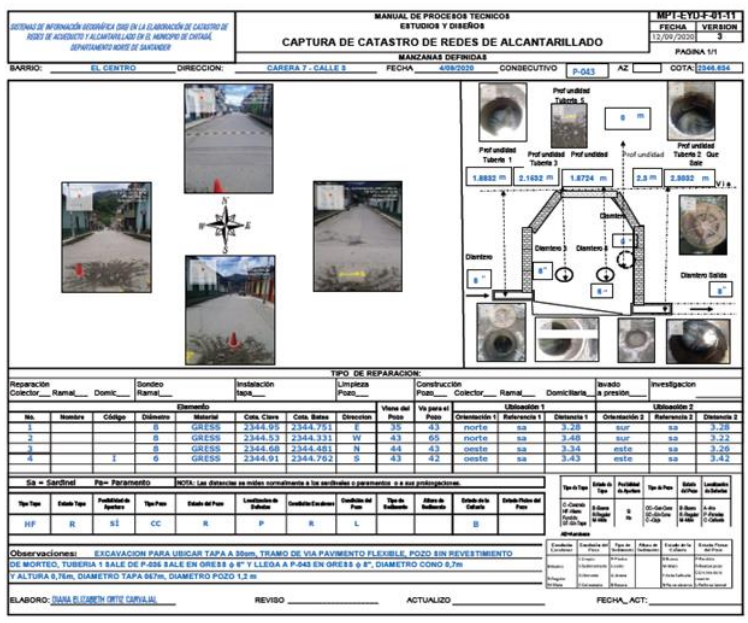

Figura 3. Ficha técnica Diligenciada.

Fuente: Elaboración propia (2021).

Después de concluida la fase de planeación en la que se diseñaron las fichas técnicas que contemplarían toda la información de los elementos que componen las redes de acueducto y alcantarillado, para el catastro de redes, se estableció la ruta más óptima en tiempo para la toma de datos en campo, en la que se siguieron las rutas de las calles y el flujo del agua residual y de la red de distribución de agua potable (Figura 4). Teniendo en cuenta lo anterior, se realizaron las visitas a los elementos en estudio, tomando los datos con excelentes prácticas ingenieriles, datos certeros, confiables y verídicos a la hora de digitarlos a las fichas técnicas.
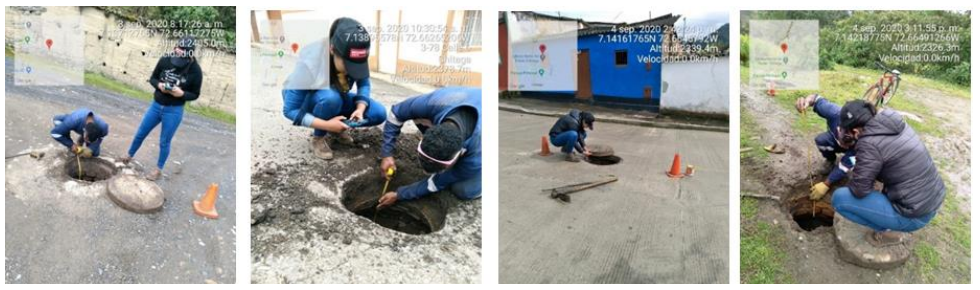

Figura 4. Recolección de Información en Campo. Fuente: Elaboración propia (2021).

Fase 3: Actualización de planos AUTOCAD Después de recoger la información en campo, se actualizaron los planos AUTOCAD de los sistemas (Figura 5). 


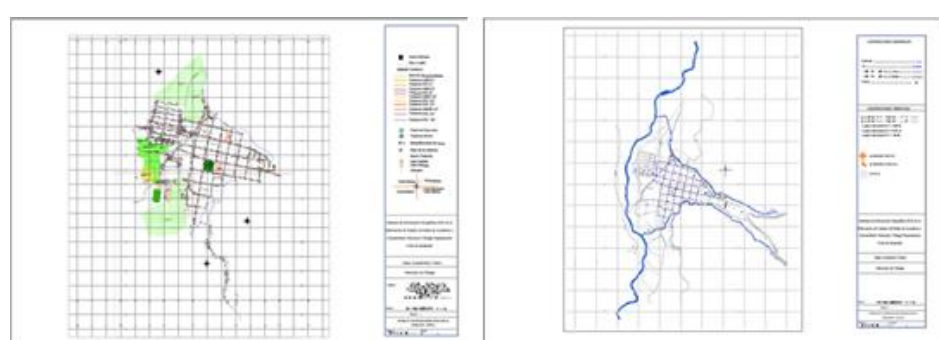

Figura 5. Planos AutoCAD Actualizados. Fuente: Elaboración propia (2021).

\section{Fase 4: Diccionarios de Datos}

Una vez digitada la información en las Fichas Técnicas de Catastro Microsoft Excel, se delimitó el sistema conceptualizando y encontrando categorías distinguidas para implementar el Sistema de Información Geográfica, que facilitaría el estado operativo de los sistemas de acueducto y alcantarillado (Figura 6).
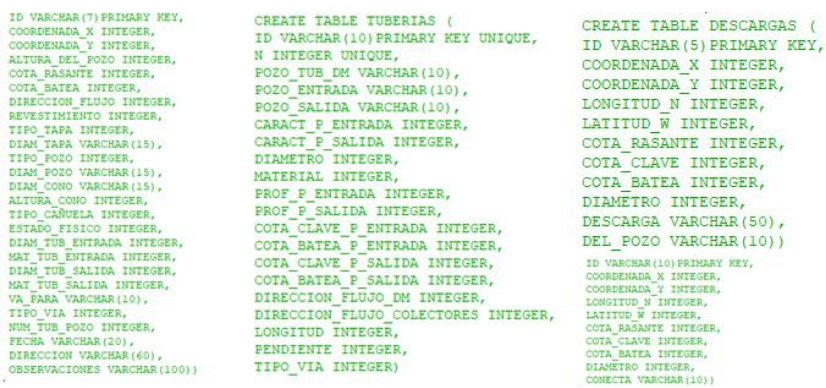

Figura 6. Categorías Diccionario de Datos para Sistema de Alcantarillado Pozos, Tuberías, Descargas y MH. Fuente: Elaboración propia (2021).

\section{Fase 5: Bases de Datos}

Antes de la creación de la base de datos, se crearon los diccionarios de datos en formato Texto Delimitado por Tabulación (TXT) que hacen más fácil su manipulación (Figura 7).

ID VARCHAR (10) PRTMARY KEY,
DIAMETRO INIEGER,
TIPO INTEGE,
ESTADO FISICO INTEGER,
OPERABILIDAD INTEGER,
RASANTE INTEGER,
VALVULA VARCHAR $(10)$,
FECHA DATETIME,
BARRIO VARCAR $(50)$,
DIRECCION VARCHAR $(50)$,
OBSERVACIONES VARCHAR $(100))$

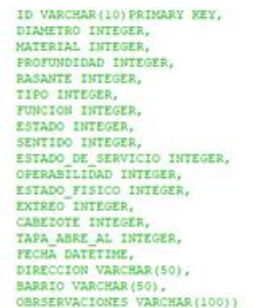

ID VARCHAR (10) PRTMARY KEY, DINMETRO INTEGER, LONGITUD INTEGER, DIRECCION VARCHAR $(100)$ )

Figura 7. Categorías de Datos para Sistema de Acueducto Hidrantes, Válvulas y Tuberías.

Fuente: Elaboración propia (2021).
Posteriormente, se creó una conexión con el Sistema Gestor de Base de Datos GeoReferenciada SQLite con lenguaje SQL para crear las bases de datos (Figura 8).

Tabla 3. Código Python Creación Base de Datos Tuberias Sistema de Alcantarillado

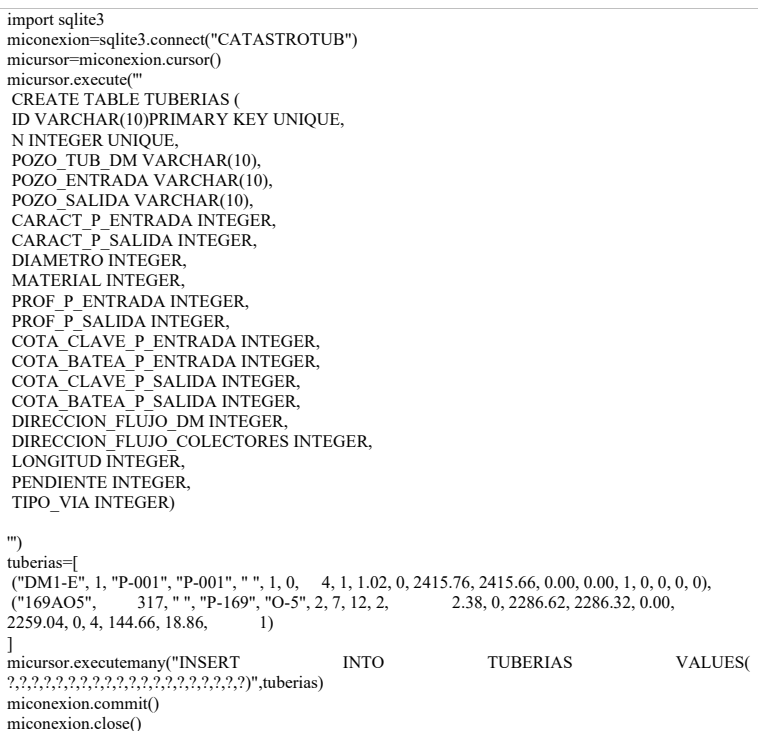

Nota: Debido a la extensión del código se presenta un resumen de 2 tuberias, la inicial y la final según diccionario de datos.

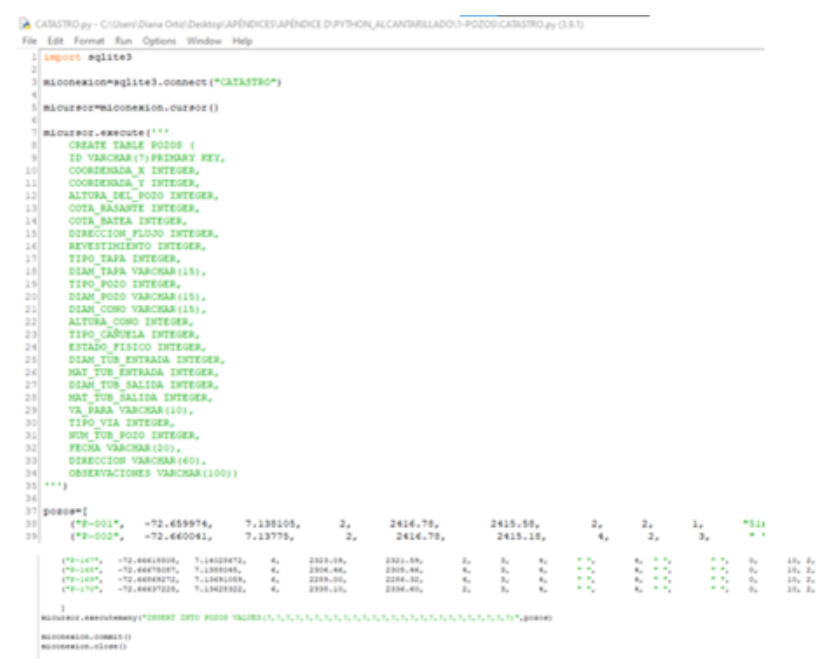

Figura 8. Creación Base de Datos Pozos de Inspección, así igual para los demás Elementos.

Fuente: Elaboración Propia (2021)

Seguidamente de crear las bases de datos, se pueden incorporar en el programa DB Browser visor del Sistema Gestor de Bases de Datos (SGBD) SQLite (Figura 9). 


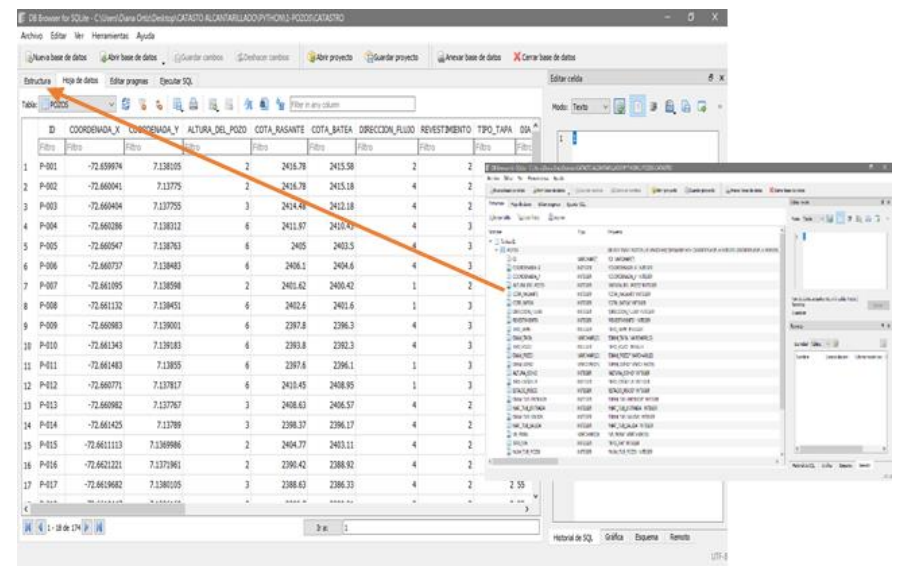

Figura 9. Estructura y Base de Datos Pozos de Inspección Sistema de Alcantarillado.

Fuente: Elaboración propia (2021)

Para los demás elementos se presenta la misma estructura.

\section{Fase 6: Implementación del SIG}

Una vez se estableció la estructura básica en tablas y se crearon las Bases de Datos con ayuda del lenguaje de programación Python, se georreferenció la información en el software principal QGIS 3.10, usando MAGNA SIRGAS datum oficial del país (IGAC, 2004).

Los archivos AUTOCAD se convirtieron a formato .DXF y con la ayuda del complemento vectorial DXF Import/Convert se montaron los planos en el SIG. Se crearon las capas correspondientes a cada sistema dependiendo de la geometría del elemento, se adjuntaron los diccionarios y bases de datos, fichas técnicas de catastro y registro fotográfico (Figuras 10-13).

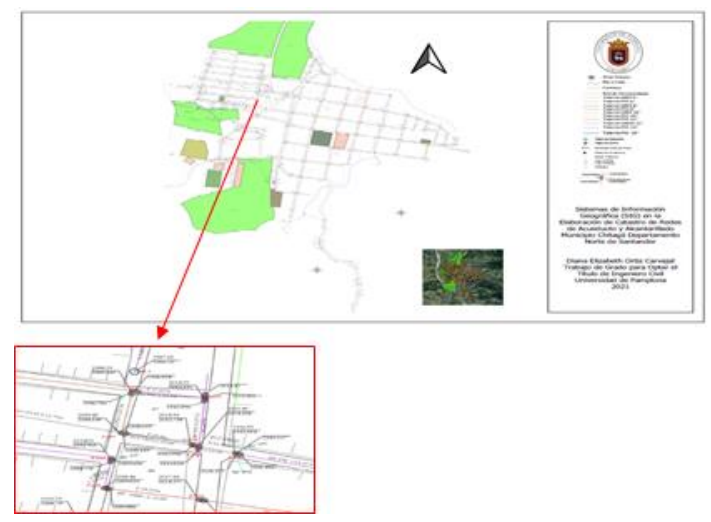

Figura 10. Plano SIG QGIS Sistema de Alcantarillado Fuente: Elaboración propia (2021)

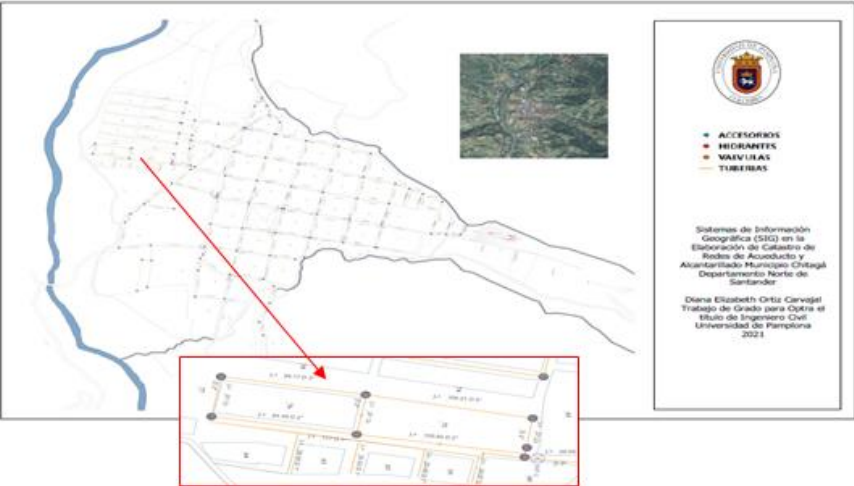

Figura 11. Plano SIG QGIS Sistema de Acueducto. Fuente: Elaboración propia (2021).

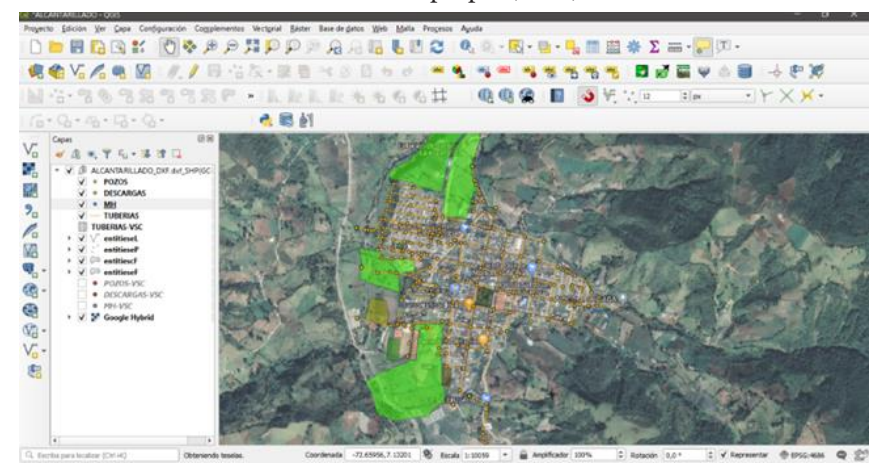

Figura 12. Sistema de Alcantarillado Municipio de Chitagá SIG QGIS.

Fuente: Elaboración propia (2021).

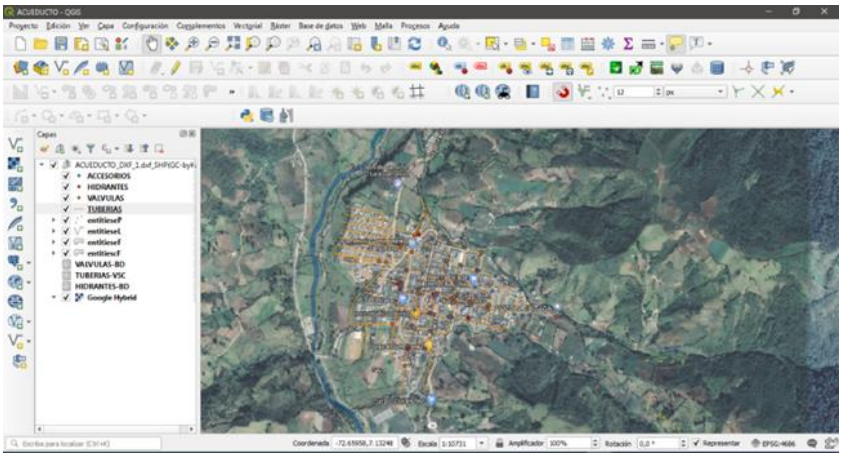

Figura 13. Sistema de Acueducto Municipio de Chitagá SIG QGIS.

Fuente: Elaboración propia (2021).

Mediante salidas opcionales y generales se presentan los resultados finales de manera textual y gráfica en el SIG (Figura 14). 


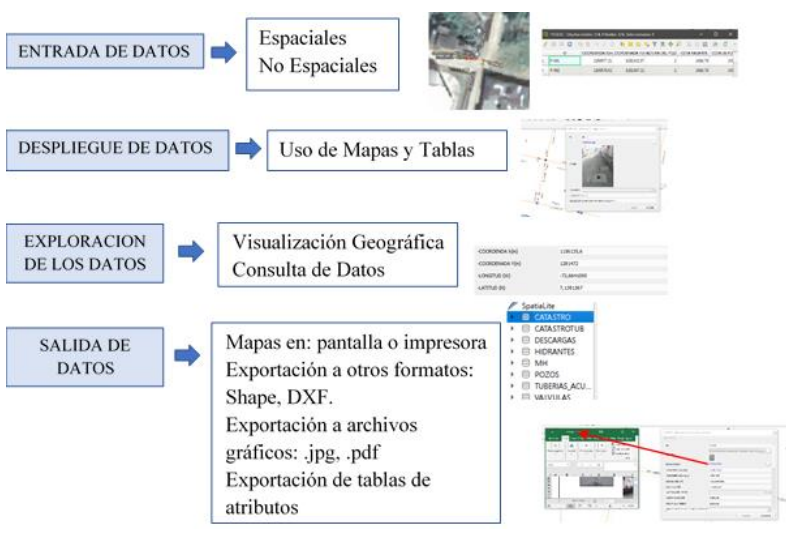

Figura 14. Funcionalidades Básicas del SIG. Fuente: Elaboración propia (2021)

Fase 7: Consultas Rápidas en las Bases de Datos Geo-Referenciadas

En esta fase se realizó un curso muy corto acerca del lenguaje SQL que fue aplicado al diseño de consultas rápidas de intervención en las bases de datos creadas sobre el SIG, para que cualquier información que se requiera de los sistemas en estudio se muestre en pantalla fácilmente y con datos certeros. El uso de comandos básicos como Select-From-Where facilitaron el diseño de consultas para los sistemas en lenguaje SQL, en las que se puede escoger datos sobre las tablas de las bases de datos (Figura 15 y 16).

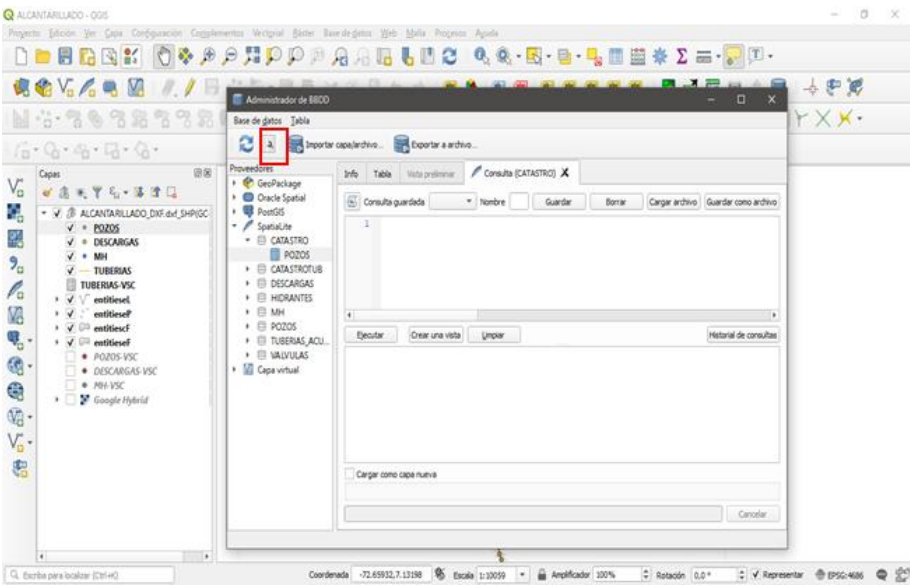

Figura 15. Ventana de Consulta Administrador BBDD QGIS Lenguaje SQL.

Fuente: Elaboración propia (2021).

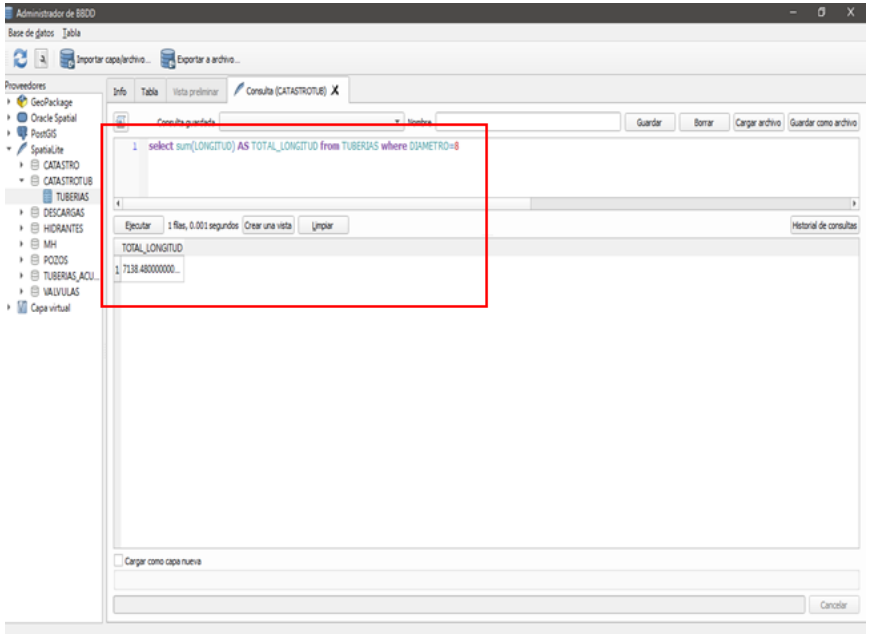

Figura 16. Consulta 4 Suma Longitud de Tuberías Diámetro 8 Plg. Fuente: Elaboración propia (2021).

Fase 8: Simulación de las redes de Alcantarillado y Acueducto del Municipio de Chitagá

A continuación, se realiza la presentación de las redes de alcantarillado y acueducto.

Simulación de la red de Alcantarillado en SWMMM

La simulación de la red en SWMM se realizó de dos formas diferentes. La primera con caudales de aguas residuales y la segunda al reconocer que el municipio de Chitagá después de la ola invernal sufrida en el año 2015, implementó alcantarillado combinado en el que en una parte del municipio se recogen y transportan las aguas lluvias gracias a estructuras como sumideros y rejillas.

Con la ayuda de SWMM (Stormwater Management Model, por sus siglas en inglés), conocido como un software de modelo dinámico de simulación de precipitaciones que trabaja con solo un acontecimiento o con un periodo extendido (EPA, 2005). Se calcularon las áreas aferentes a cada colector, la población servida y seguidamente el caudal doméstico. Se dibujaron las tuberias y nodos (pozos) con sus respectivas propiedades, en cuanto al alcantarillado pluvial se utilizaron datos de curvas IDF para calcular un Hietograma de Frecuencia para un periodo de retorno, sobre el mismo modelo sanitario 
se dibujaron las subcuencas que simularían el fenómeno (Figuras 17 y 18).

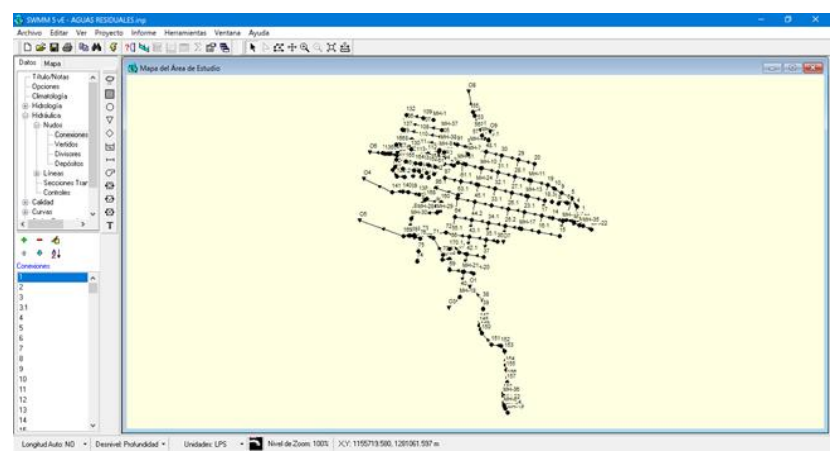

Figura 17. Plano Red Alcantarillado Sanitario en el Software SWMM.

Fuente: Elaboración propia (2021).

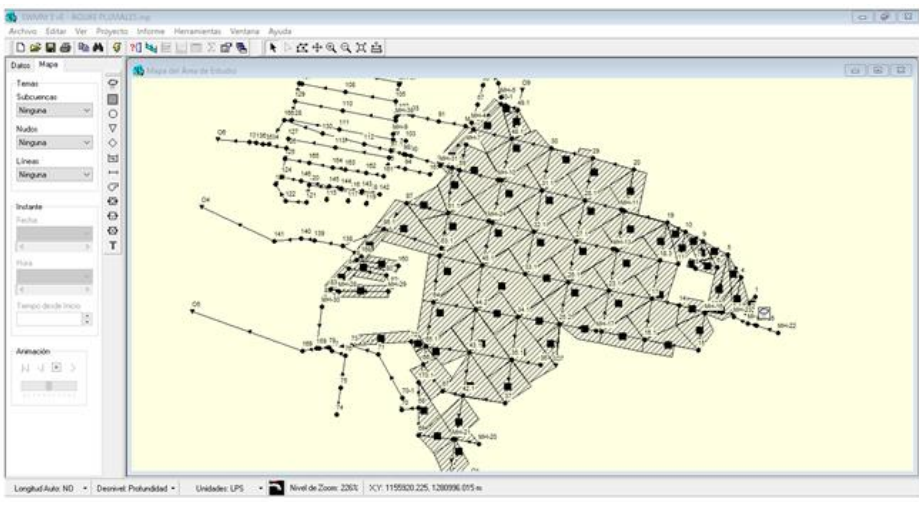

Figura 18. Plano Alcantarillado Combinado Software SWMM. Fuente: Elaboración propia (2021).

Simulación de la red de acueducto en EPANET

Para establecer la simulación de la red de distribución de acueducto, se exportó de AutoCAD el trazado del sistema al software EpaCAD con el fin de crear las tuberías y los nodos con dimensiones reales. Posteriormente, el archivo es guardado en el formato de EPANET, para luego ser procesado en el software. La simulación de la red en EPANET se realizó asumiendo un dato de presión, dado que en la planta de tratamiento no existe medición de esta. El funcionamiento de la planta se comparó con un embalse que funciona las 24 horas al día ya que la distribución no es un sistema intermitente. Seguido del montaje de la red a EPANET se disponen las características hidráulicas en el software, para establecer las ecuaciones de cálculo permitidas en un sistema de acueducto.
Como en el municipio no se tiene una curva de consumo se establece una demanda a cada nodo base derivada del cálculo del caudal por el método de Áreas.

Para cada uno de los nodos creados en el software, se ingresaron los datos de la cota terreno para las tuberías y los siguientes datos, el coeficiente de rugosidad del material (PVC) usada la ecuación de Darcy-Weisbach con valor de 0,0015 y el diámetro interno establecido según la resolución 0330 de 8 de junio de 2017 y el RDE establecido para la presión máxima a soportar por las tuberías.

De igual manera se establecieron en la red las válvulas de regulación que garantizan el servicio del sistema cuando hay fugas o reparaciones y las válvulas reductoras de presión encontradas en el terreno. Se hizo la disposición de los hidrantes de la zona de estudio, teniendo en cuenta el caudal a servir según la población y el uso establecido por la Resolución 0330 del 8 de junio de 2017 (Figura 19).

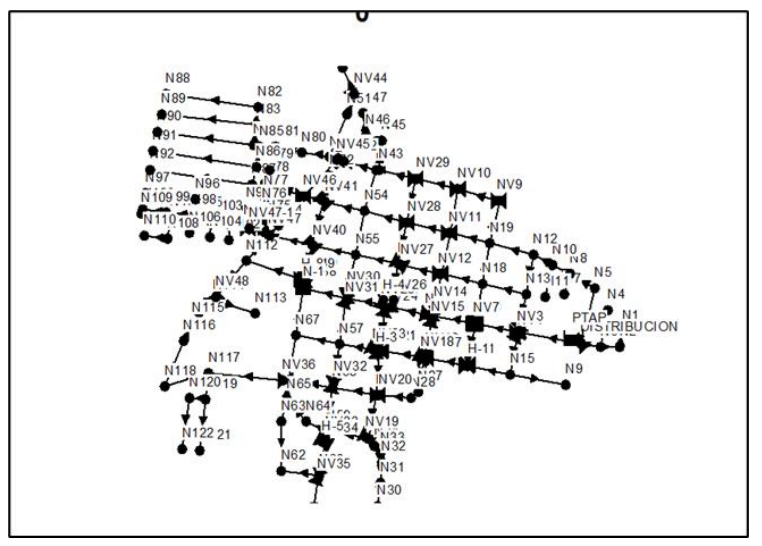

Figura 19. Plano sistema Acueducto EPANET. Fuente: Elaboración propia (2021).

Fase 9: Interfaz DEOC

Mediante el lenguaje de programación Python se diseñaron 8 interfaces gráficas, 4 para el sistema de alcantarillado (POZOS, TUBERIAS, DESCARGAS y $\mathrm{MH}$ ) y 3 para sistema de acueducto (TUBERIAS, VÁLVULAS e HIDRANTES) y una más que integra la totalidad de ellas. Esto se logró con la ayuda de las librerías Tkinter y Sqlite3, mediante código se diseñaron las raíces de interfaz, icono (deoc), título de la 
interfaz (CATASTRO DE REDES CHITAGA), frames, labels, funciones, creación de la base de datos, excepciones, menús desplegables, ventanas emergentes de información, de advertencia y de abrir ficheros, entrys, áreas de texto, barra de desplazamiento scrooll y por último buttons.

La interfaz presenta menús desplegables que permiten ejecutar las diferentes funciones; tiene icono y titulo identificativos; permite combinar colores en textos y en áreas de texto; el campo Id es aquel mediante el cual se realizaran las operaciones CRUD que deberá ser digitado por el usuario (Figuras 20-22).

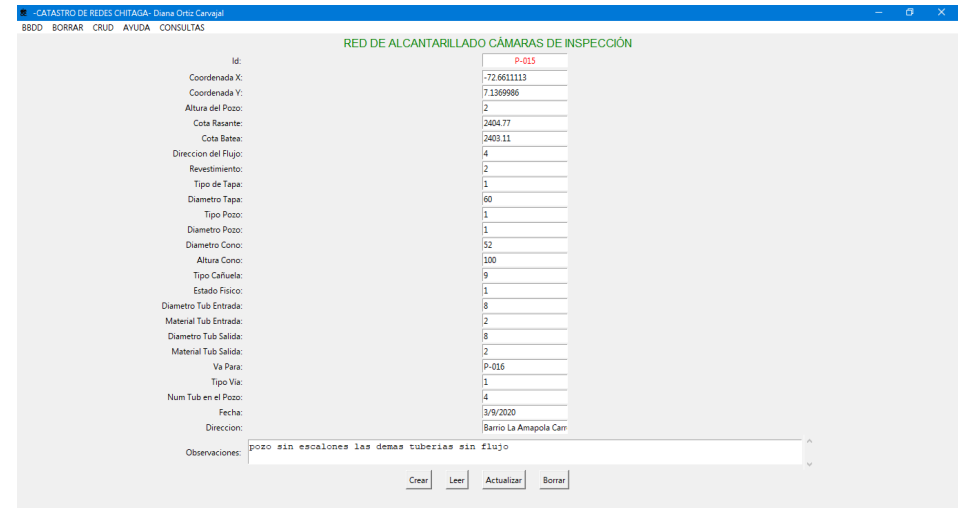

Figura 20. Interfaz de Consulta Catastral. Fuente: Elaboración propia (2021).

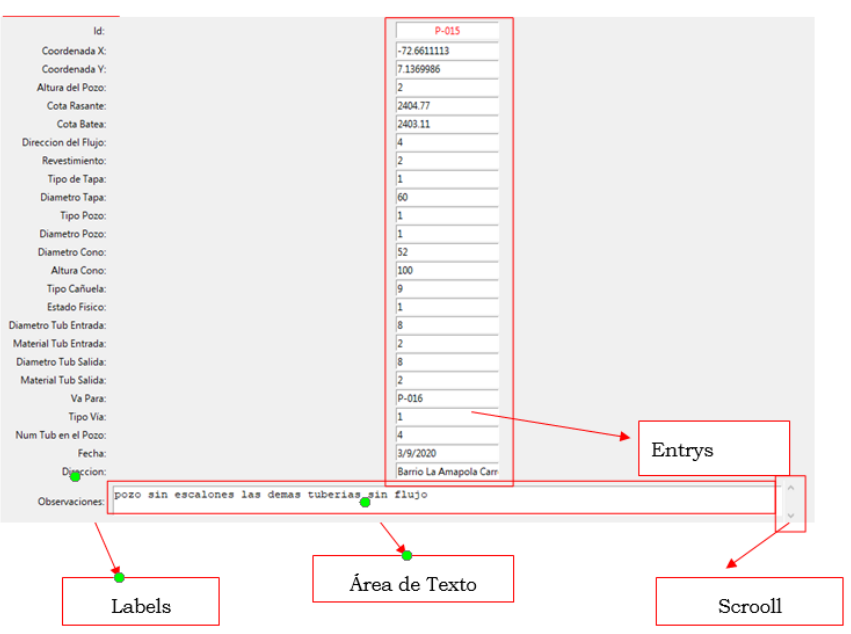

Figura 21. Componentes de la Interfaz. Fuente: Elaboración propia (2021).

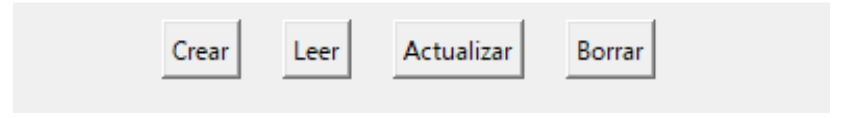

Figura 22. Buttons.

Fuente: Elaboración propia (2021).
Esta ventana se crea cuando la base ya ha sido conectada o creada y se pretende volver a crear (Figuras 23 y 24 ).

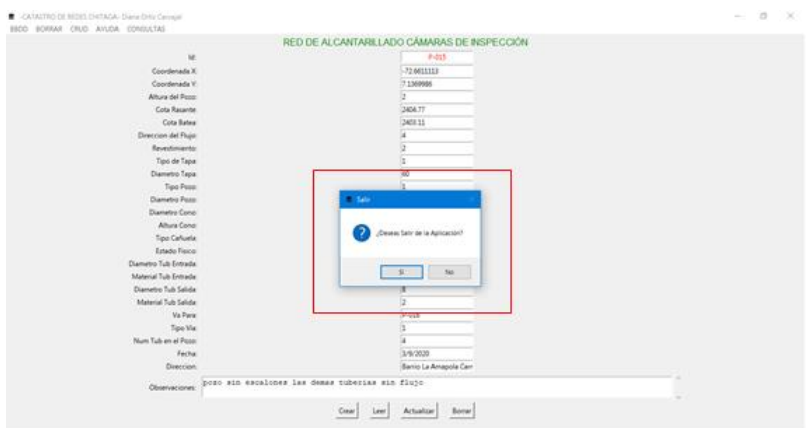

Figura 23. Ventana de Creación de BBDD

Fuente: Elaboración propia (2021).

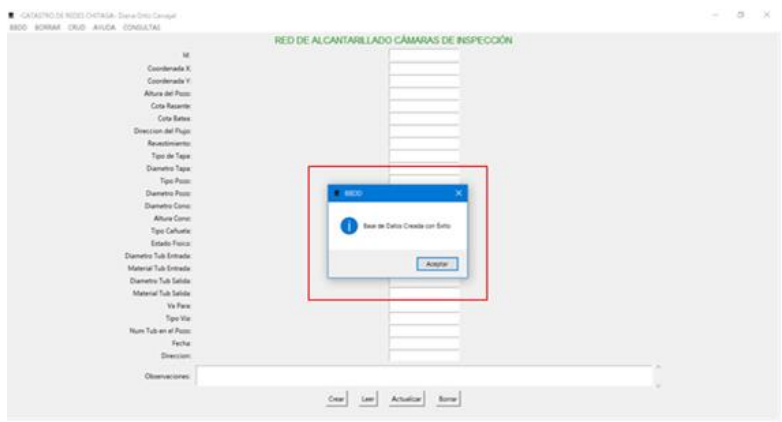

Figura 24. Ventana Emergente de Advertencia. Fuente: Elaboración propia (2021).

Ventana emergente que permite al usuario confirmar si desea salir de la Aplicación (Figura 25).

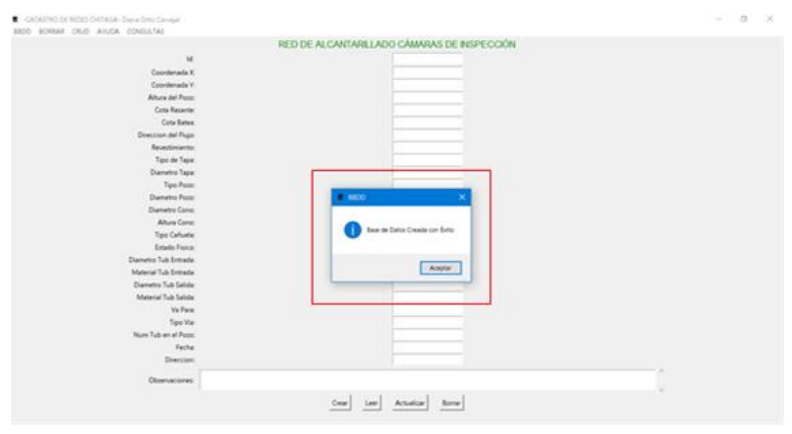

Figura 25 Ventana Emergente Question. Fuente: Elaboración propia (2021) 


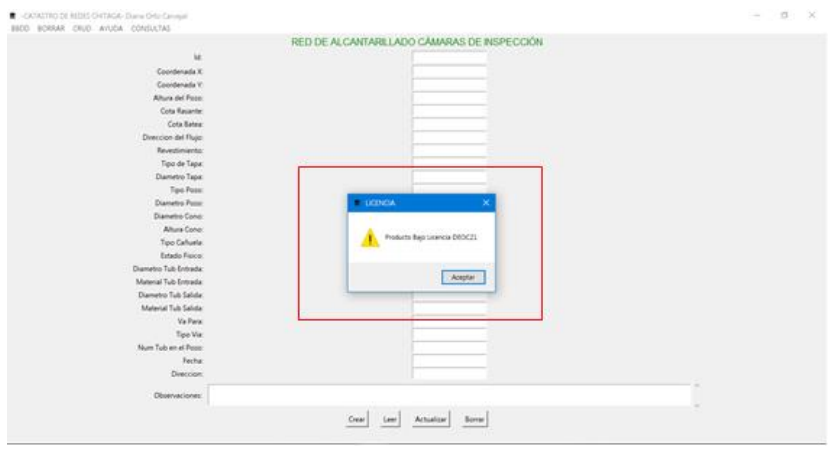

Figura 26. Datos de Propiedad (Licencia).

Fuente: Elaboración propia (2021)

La interfaz permite mediante el menú BBDD y el submenú Conectar crear la base de datos para cada elemento, cuya base de datos gracias al menú CRUD o a los botones Crear, Leer, Actualizar o Borrar, podrá ser manipulada por el usuario, es decir, crear registros nuevos, leer los existentes, actualizar algún campo de algún registro o borrar registros si así se desea.

Con el menú BORRAR, permite Borrar los Campos en pantalla por si se va realizar otra operación. El menú AYUDA, muestra las diferentes ventanas que se pueden crear en una interfaz y algunos datos de Propiedad del Estudiante. El menú CONSULTAS permite conocer la ruta de un Archivo que contenga alguna consulta rápida.

Para aspectos relacionados con el lenguaje de programación Python usados como referencia en el estudio se consideraron a Celi, (2013); Fitzcarrald, (2015); Gómez, (2007); Jiménez y Aviles, (2019).

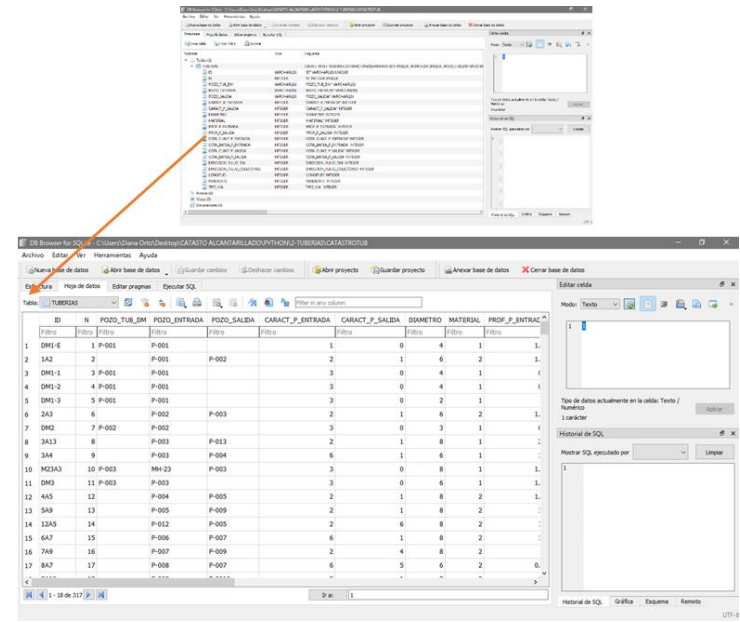

Figura 27. Estructura y Base de Datos Tuberías Sistema de Alcantarillado.

Fuente: Elaboración propia (2021)

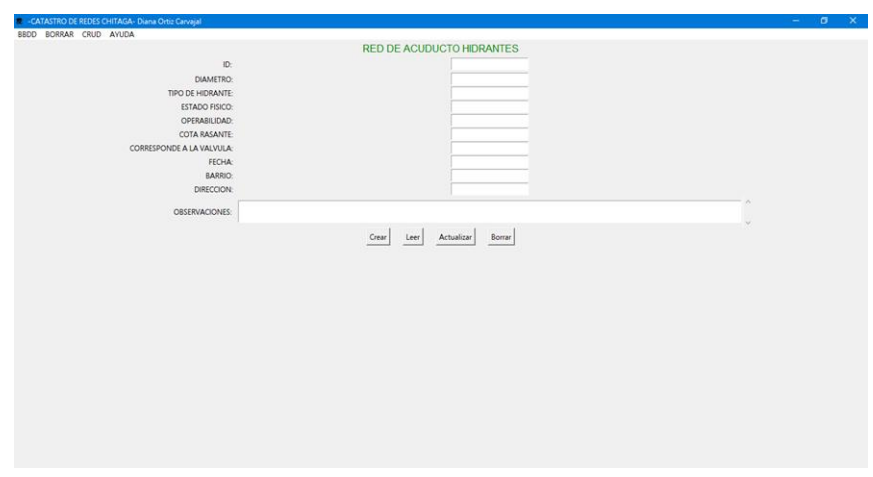

Figura 28. Interfaz DEOC Hidrantes. Fuente: Elaboración propia (2021)

La evaluación inicial de los Sistemas de Acueducto y Alcantarillado del Municipio de Chitagá evidenció que la información se encontraba en forma dispersa, existían modelos de conducta coligados a la ausencia de direccionamiento, gestión y control de la operatividad de los mismos por parte de la secretaría de Servicios Públicos y Domiciliarios, presentando dificultad de manejo y análisis. Con el cumplimento de este trabajo esta información fue digitalizada, analizada, centralizada y clasificada.

Dado que es un proyecto que respeta a una base de datos de información geográfica gestionada en QGIS, su importancia, su uso y eficiencia dependen primordialmente del comportamiento de sus gestores, quienes deberán mantenerla y actualizarla siempre que sea necesario, lo que permite resultados de su uso más gratificantes en cuanto a administración, gestión y funcionamiento. La base de datos fue planeada y fundada a partir de las insuficiencias actuales y permisibles del municipio. La información de todo el sistema y sus elementos se maneja de manera digital, actualizando permanentemente cualquier cambio o intervención sobre el mismo, producto de órdenes de trabajo o reparaciones, garantizando su disponibilidad en todo momento y conservación. Así mismo, se ha convertido en una herramienta importante de administración de los activos que hacen posible la operación del sistema y permiten su ubicación en un entorno geográfico.

Como se mencionó anteriormente, la información relaciona con cada el elemento en la realidad, es identificado como un objeto en el SIG con 
atributos y propiedades, lo que le permite conocer a los gestores del municipio la información relacionada con él, incluso en tiempo real. La base de datos puede ser aumentada de acuerdo a requisitos de los administradores, inclusive vinculada con otras bases de datos como censos o usuarios.

En cuanto a la relación costo beneficio, resulta importante mencionar los costos que representa la implementación de la propuesta. El uso de QGIS software motor de SIG de libre acceso y sin costo alguno obedece a las potentes, llamativas, precisas, vistosas, y de calidad funcionalidades que este ofrece, así como la operación del SIG. Los costos para el desarrollo del presente proyecto, hacen más factible la implementación del SIG para la gestión del acueducto y del alcantarillado objeto de estudio.

En términos generales, la implementación del SIG aquí propuesta, supone una mejoría en algunos de los procesos que actualmente llevan a cabo los administradores de los sistemas de acueducto y alcantarillado; esto también abre muchas opciones enfocadas a trabajos de gestión, mantenimiento o mejoras de estas obras civiles.

\section{Referencias Bibliográficas}

Avendaño, Z., A. Z., y Cadena M., Y. K. (2014). Uso de Sistemas de Información Geográfica en la Determinacion de Amenazas por Inundación en el Municipio de Chía (Tesis de pregrado). Universidad Católica de Colombia, Bogota, Colombia. https://repository.ucatolica.edu.co/ handle/10983/1569

Biblioteca Virtual, Biblioteca Luis Ángel Arango. (11 de 02 de 2021). Sistema de Información Geográfica. Bogotá. Obtenido de http://www. banrepcultural.org/blaavirtual/ayudadetareas/ geografica/geo42.htm

Biere, R. y Aitziber, E. (2010). Herramienta para el diagnostico de la accesibilidad en entornos de patrimonio historico, en base a escaneado láser y realidad virtual. ACE Architecture, City and Enviroment (Arquitectura Ciudad y Entorno), 61-90.
Borcosque, J. (1997). Apuntes sobre sistemas de Información Geográficos. Santiago de Chile: Departamento de Ingeniería Geográfica. Universidad de Santiago de Chile.

Buitrago, P., S. A. (agosto de 2018). Propuesta de Implementación de un SIG para la Administración y Gestión del Sistema de Acueducto del Municipio de Bagía Solano Chocó (Tesis de pregrado). Universidad Militar Nueva Granada, Bogotá, Colombia. https://repository. unimilitar.edu.co/handle/10654/18099

Celi, M. S. (2013). Control de un generador de pulsos, una fuente de voltaje-corriente y matriz por medio del protocolo GPIB programado en Python (Tesis de pregrado). Universidad San Francisco de Quito, Quito, Ecuador. https:// repositorio.usfq.edu.ec/handle/23000/2749

Cesario, L. (1986). Denver's Mapping Information Management System. AWWA Annual Conference. Denver Colorado (USA), 669-681.

Cualla, R. A. (1995). Elementos de diseño para acueductos y alcantarillados. Bogota: Escuela Colombiana De Ingeniería.

Departamento Nacional de Planeación Financiera de Desarrollo Territorial. (1997). Análisis del sector de agua potable y saneamiento en Colombia. Comisión Reguladora de Agua. https://www.paho.org/col/dmdocuments/ analisis_sectorial_aguaysanea_colombia.pdf

Diaz, M., D. B. y Mercado Y., N. E. (2016). Gestión de Redes de Acueducto del Municipio de San Juan Nepomuceno Bolívar Mediante la Implementación de Herramientas SIG. (Tesis de posgrado). Universidad de Manizales, Manizales, Colombia. http://ridum.umanizales.edu.co/ xmlui/handle/20.500.12746/2886

Empresas Públicas de Medellín. (2021). EPM. Obtenido de EMP: https://www.epm.com.co/ site/

EPA. (2005). Manual del Usuario SWMM.

EPM. (Versión 08). Instructivo para referenciación de los sistemas de acueducto y alcantarillado. En E. P. EPM. Medellín.

ESRI. (2016). ArcGis for Desktop. http://desktop. arcgis.com/es/arcmap/10.3/manage-data/ gdb-architecture/the-geodatabase-is-object- 
relational.htm

Fitzcarrald, A. (2015). Aplicación de un Sistema de Información Geográfico para el monitoreo de las condiciones oceanográficas del Fenómeno del Niño (Tesis de posgrado). Marina De Guerra Del Perú Escuela Superior De Guerra Naval, La Punta, Perú. https://aquadocs.org/handle/1834/8156

Fragoso, L., Ruiz, J. R., y Juárez, A. B. (2013). Sistema para control y gestión de redes de agua potable de dos localidades de México. Ingeniería Hidráulica y Ambiental, 34(1), 112-126.

Gobernación de Norte de Santander- DAG Ingeniería. (2014). Contratación de una consultoría especializada para la actualización de los indicadores y la generación de un diagnóstico técnico de la línea base de la infraestructura en la prestación de servicios públicos, para el departamento Norte de Santander. Informe diagnóstico - municipio de Chitagá: Secretaría Agua Potable y Saneamiento Básico.

Gómez, M. A. (2007). Arquitectura y metodología para el desarrollo de sistemas educativos basados en videojuegos (Tesis de doctorado). Universidad Complutense de Madrid, Madrid, España. https://eprints.ucm.es/id/eprint/8198/1/T30447. pdf

Gómez, A. A., Velasquez, M. J., y Rodriguez, J. J. (2006). Levantamiento catastral diagnóstico, inspección para la actualización de redes de acueducto en las zona 9a, 9b, 9c de la ciudad de Armenia (Tesis de pregrado). Universidad del Quindio, Armenia, Colombia. https://bdigital. uniquindio.edu.co/bitstream/handle/001/5907/ TESIS.pdf

Google Earth. (13 de 02 de 2021). Localización Municipio de Chitagá. Obtenido de Google Earth Web: https:// earth.google.com/web/@7.13566654,72.66493094,2332.45094691a,3558.93966546d,3 5y,105.28130077h,16.55606079t,0r

Gulf of California Marine Programa. (11 de 02 de 2021). Importancia de los Sistemas de Información Geográfica (SIG) en la Conservación. Obtenido de http://gulfprogram. ucsd.edu/blog/coastal-and-marine/importanciade-los-sistemas-de-informacion-geografica-sigen-la-conservacion/
Guzmán, J. S. (2021). Elaboración del Catastro de la Red de Distribución de Agua Potable del Municipio de Susacón en el Departamento de Boyacá. Susacón en el Departamento de Boyacá (Tesis de pregrado). Universidad Santo Tomas, Bogotá, Colombia. https://repository.usta.edu. co/handle/11634/33934

Healey, R. (2001). Database management systems. Longley, Goodchild.

Hernández, J. A. y Osorio, S. S. (2019). Diseño hidráulico de la primera fase de la red de alcantarillado del casco urbano del municipio de Chipaque (Tesis de posgrado). Universidad Católica de Colombia, Bogotá, Colombia. https:// repository.ucatolica.edu.co/handle/10983/23874 IGAC, I. G. (1998). Principios básicos de cartografía temática Nancy Aguirre Gutiérrez (Investigación y Coordinación). Instituto Geográfico Agustín Codazzi, IGAC Ministerio de Hacienda y Crédito Público.

IGAC. (2004). Instituto Geográfico Agustín Codazzi. Obtenido de https://www.igac.gov.co/ Jaimes, L. M. (2019). Elaboración del Catastro de la Red de Alcantarillado Implementando Sistemas de Información Gepgráfica en el Municipio de Mutiscua Norte de Santander (Tesis de pregrado). Universidad de Pamplona, Pamplona, Colombia.

Jiménez, E. A. y Aviles, J. (2019). Análisis de la red social Twitter para la identificación de patrones que generan oportunidades de negocio en la ciudad de Guayaquil utilizando el entorno de trabajo Jupyter Notebook y el lenguaje de programación Python (Tesis de pregrado). Universidad de Guayaquil, Guayaquil, Ecuador:. López, H. T. (2012). Sistema de Información Geográfica aplicado al Catastro de Agua Potable del Cantón Paute, Ecuador (Tesis de maestría). Universidad San Francisco de Quito, Quito, Ecuador. https://repositorio.usfq.edu.ec/ handle/23000/2013

Martínez, G. (2013). Sistemas urbanos de drenaje sostenible" SUDS" como alternativa de control y regulación de las aguas lluvias en la Ciudad de Palmira (Tesis de pregrado). Universidad Militar Nueva Granada, Bogotá, Colombia. https://repository.unimilitar.edu.co/ 
handle/10654/11075

Ministerio de Vivienda. (2017). Reglamento Técnico De Agua Del Sector De Agua Potable Y Saneamiento Básico RAS. Ministerio de Vivienda, Colombia. https://www.minvivienda.gov.co/ viceministerio-de-agua-y-saneamiento-basico/ reglamento-tecnico-sector/reglamento-tecnicodel-sector-de-agua-potable-y-saneamientobasico-ras

Morales, D. A., y Rodríguez, M. A. (2016). Catastro técnico y modelación hidráulica del sistema de alcantarillado combinado del centro urbano de la parroquia Lloa del Distrito Metropolitano de Quito con EPA-SWMM V5.1.010 (Tesis de pregrado). Pontificia Universidad Catolica de Ecuador, Quito, Ecuador. http://repositorio.puce. edu.ec/handle/22000/12302

Moran, M. y Malma, J. D. (2013). Sistema de Información Geográfico con interfaz web de la ciudad de Puno (Tesis de pregrado). Universidad Nacional del Altiplano, Puno, Perú. http:// repositorio.unap.edu.pe/handle/UNAP/2374

Muñoz, A., Roncancio, C. J., y Mendoza, S. A. (2018). Implementación de un Sistema de Información Geográfica para las Plantas de Tratamiento de Agua Potable y Residual de la Armada Nacional de Colombia (Tesis de pregrado). Universidad Nacional Abierta y a Distancia, Bogotá, Colombia. https://repository. unad.edu.co/handle/10596/17930

Orjuela, F. J. (2018). Incidencias de la informacion catastral en la ampliación de servicios públicos, sector Playitas (Tesis de maestría). Universidad Nacional de Colombia, Arauca, Colombia.https:// repositorio.unal.edu.co/handle/unal/76828

Palacio, A. V. (2017). Implementación de sistemas de información geográfica en la gestón de espacios protegidos (Tesis de doctorado). Universitat Rovira i Virgili, España. https://www. tdx.cat/handle/10803/586066

Pérez, D., M. (2014). Extensión de Aplicaciones mediante Python embebido. (Tesis de pregrado). Escuela Técnica Superior de Ingeniería y Sistemas de Telecomunicación, España. http://oa.upm. es/44475/

Rodriguez, A., M. L. y Rodriguez D., J. F. (2014). Modelación y evaluación hidráulica del alcantarillado del municipio de chocontácundinamarca, mediante el uso del software epa swmm (Tesis de pregrado). Universidad Catolica de Colombia, Bogotá, Colombia. https:// repository.ucatolica.edu.co/handle/10983/1686 Rossiter, D. G. (1994). Special Topics in Soil, Crop and Atmorpheric Siences. Land Evaluation, with emphais on computer application: http:// www.css.cornell.edu/faculty/dgr2/_static/files/ Scas494/s494ch1.pdf

Sabogal, Z. y Rincón, A. (2017). Administración de datos generados por un catastro de redes por medio de herramientas SIG (Tesis de pregrado). Universidad de Manizales, Manizales, Colombia. http://ridum.umanizales.edu.co/xmlui/ handle/20.500.12746/3240

Sánchez, I. Y. y Amorós, M. I. (2012). Gestion de acueducto y alcantarillado mediante sistemas de información gográfica. ING. HIDRÁULICA Y AMBIENTAL, 33(3).

Santiago, O. A. (2010). Desarrollo de una metodologia para el levantamientp del catastro de acueducto (Tesis de pregrado). Universidad de los Andes, Bogotá, Colombia. https://repositorio. uniandes.edu.co/bitstream/handle/1992/14493/ u419349.pdf

Sarría, F. A. (2010). Sistemas de Información Geográfica. En F. A. Sarría, Sistemas de Información Geográfica (pág. 7).

SENA-Servicio Nacional de Aprendizaje. (2005). Fontanería Municipal. Sistemas de Acueductos. Bogotá, Colombia: Ministerio de Ambiente. https://repositorio.sena.edu.co/sitios/ calidad_del_agua/fontaneria_municipal/pdf/ FONTANERIA\%20MUNICIPAL.pdf

Solano, D. F. (2002). Aplicación de los Sistemas de Información Geográfica a la gestión técnica de redes de distribución de agua potable. Valencia, España: Universidad Politécnica de Valencia.

Torres, W. E. (2015). Implementación de un sistema de información geográfica en la unidad de análisis del departamento de seguridad de naciones unidas para Colombia (Tesis de pregrado). Universidad Distrital Francisco José de Caldas, Bogotá, Colombia. http://repository. udistrital.edu.co/handle/11349/3052

Torres, V., P. A., y Vélez G., V. H. (2010). 
Diagnóstico y actualización del catastro de las redes matrices de acueducto del municipio Dosquebradas Risaralda (Tesis de pregrado). Universidad Libre, Pereira, Colombia. http:// repository.unilibre.edu.co/handle/10901/17044 Zapata, M. V., Martinez, C., y Gonzalez, C. (s.f.). Implementación de un Sistema de Información Geografica, SIG, en el Parque Nacional Natural Farallones de Cali. Revista Ingeniería e Investigación, (43), 25-29. https://revistas.unal. edu.co/index.php/ingeinv/article/view/21077 Zarzosa, N. L., y Andrés, M. N. (2004). Sistemas de información geográfica. Practicas con ArcView. Catalunya: Universidad Politécnica de Catalunya. 


\section{Anexos}
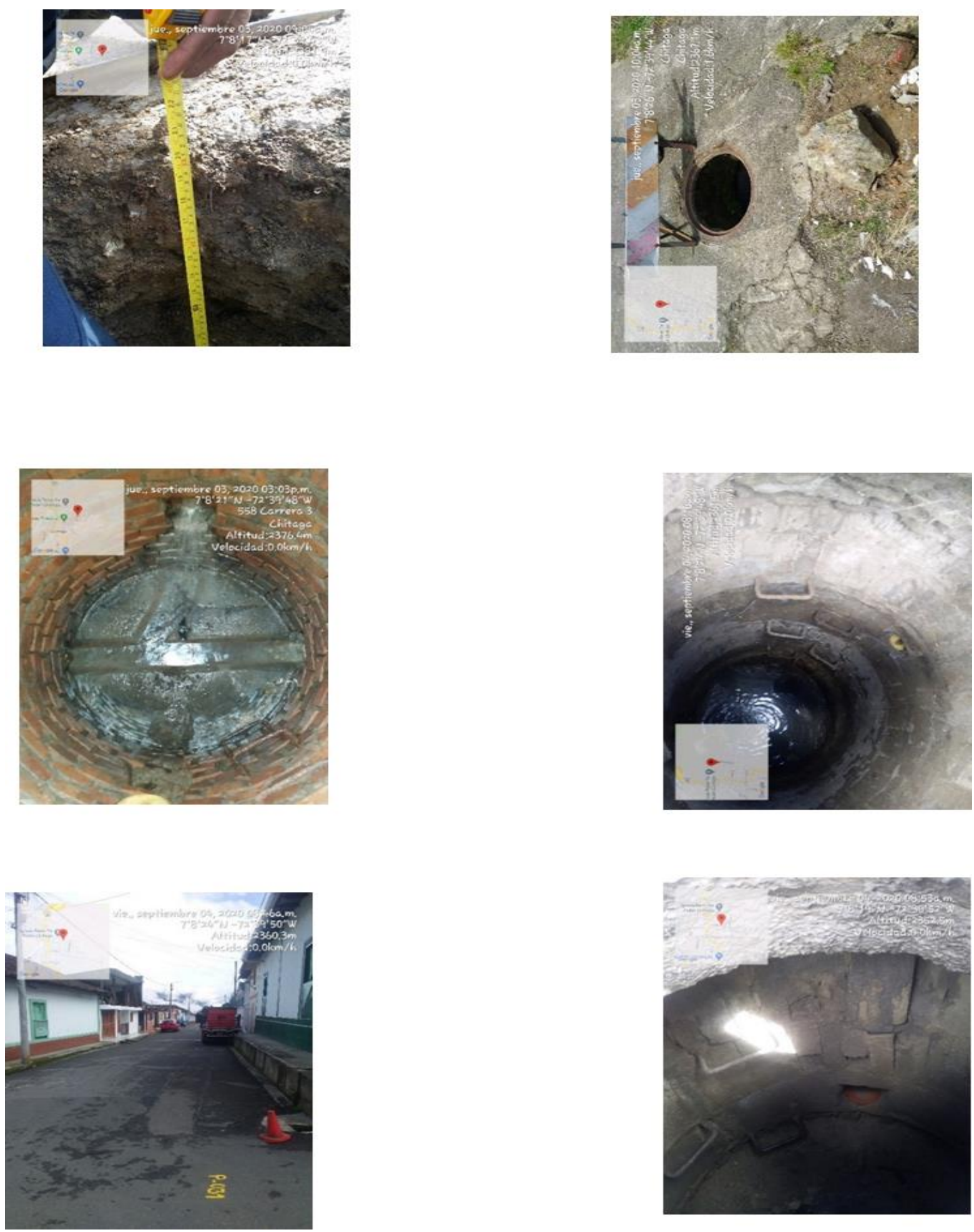

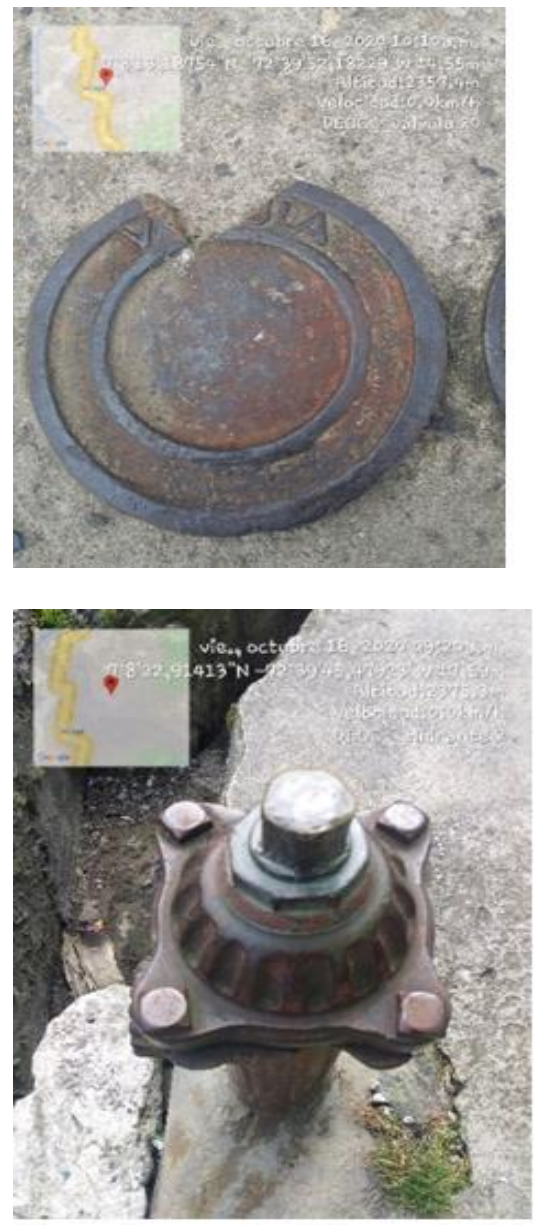
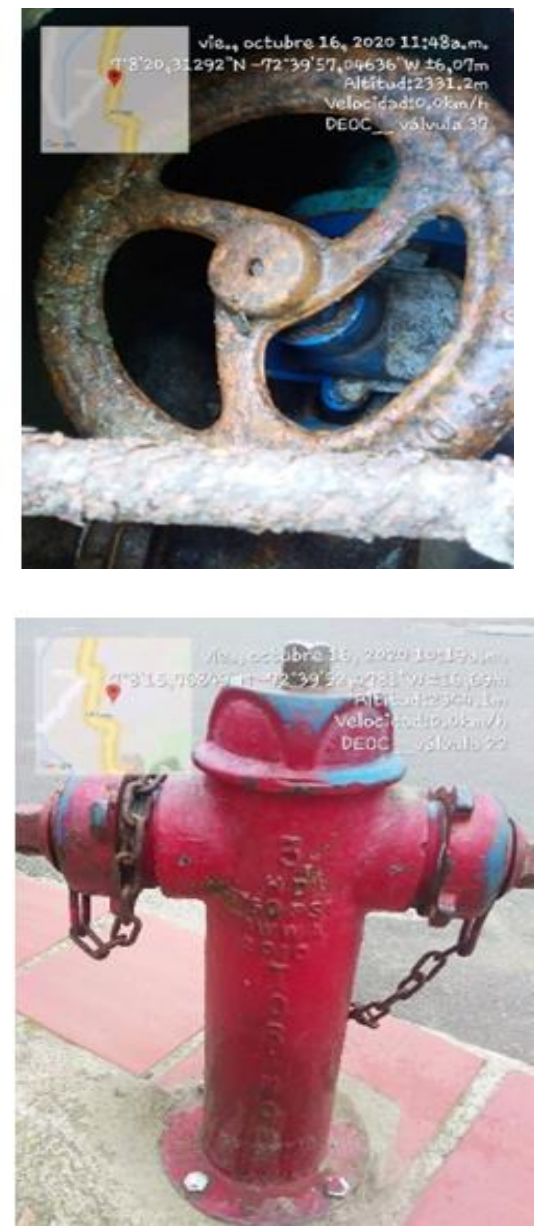